\title{
Entrelacs
}

Cinéma et audiovisuel

\section{Alejandro Jodorowsky, d'un art à} l'autre.

Connexions, circulations, hybridations

Yoann Hervey, Fabien Meynier et Céline Saturnino

\section{OpenEdition}

\section{Journals}

Édition électronique

URL : http://journals.openedition.org/entrelacs/5741

DOI : 10.4000/entrelacs.5741

ISSN : 2261-5482

Éditeur

Éditions Téraèdre

Référence électronique

Yoann Hervey, Fabien Meynier et Céline Saturnino, « Alejandro Jodorowsky, d'un art à

l'autre. », Entrelacs [En ligne], 16 | 2019, mis en ligne le 06 novembre 2019, consulté le 04 décembre 2019. URL : http://journals.openedition.org/entrelacs/5741 ; DOI : 10.4000/entrelacs.5741

Ce document a été généré automatiquement le 4 décembre 2019

Tous droits réservés 


\title{
Alejandro Jodorowsky, d'un art à l'autre.
}

\author{
Connexions, circulations, hybridations
}

Yoann Hervey, Fabien Meynier et Céline Saturnino

1 Alejandro Jodorowsky est essentiellement reconnu en tant que cinéaste et scénariste culte de bandes dessinées depuis la réalisation de ses deux films El Topo (1971) et La Montagne sacrée (La Montaña sagrada, 1973) ainsi que l'écriture de la série L'Incal, en collaboration avec le dessinateur Mœbius, dont le premier tome parait en 1981 aux Humanoïdes Associés. Il est pourtant l'auteur d'une œuvre abondante et protéiforme qui, loin de se limiter au cinéma ou à la bande dessinée, comprend une grande pluralité de pratiques artistiques.

2 Originaire du Chili, il débute par le théâtre de marionnettes qui le mène en France en 1954 où il s'adonne à la pantomime avec le Mime Marceau pour lequel il écrit des mimodrames ${ }^{1}$. Son goût pour le spectacle vivant s'affirme et se concrétise lors de la création avec, notamment, Fernando Arrabal et Roland Topor de l'« anti-mouvement Panique ». Ce collectif visait à bousculer les conventions théâtrales en privilégiant l'acte performatif avec ce que cela implique d'aléatoire, d'improvisation et d'extravagance ${ }^{2}$. Dans cette période qui s'étale sur une dizaine d'années (1962-1974), il met en scène plus de trois cents spectacles entre Paris et Mexico. Parallèlement, il fait ses premiers pas au cinéma en adaptant une pièce d'Arrabal, Fando et Lis (Fando y Lis, 1968), mais ce sont ses deux films suivants, El Topo et La Montagne sacrée, réalisés au Mexique, qui lui apportent une renommée en tant que cinéaste et le rattachent aux mouvements contre-culturels des années 1970. À partir des années 1980, Jodorowsky diversifie ses activités artistiques. Il essuie des échecs cinématographiques mais s'impose comme un scénariste incontournable de bandes dessinées, avec des séries telles que L'Incal, Les Aventures d'Alef-Thau ou encore La Caste des Méta-Barons ${ }^{3}$ qui toutes questionnent les tentations autoritaires des sociétés en accordant une place centrale aux violences et aux transformations que cela provoque sur les corps mêmes des individus. Il se tourne également vers la littérature où il crée là encore une œuvre dense faite de romans (parmi lesquels Le Paradis des perroquets et L'Arbre du dieu pendu ${ }^{4}$ ), 
de recueils de nouvelles et de poésie (en particulier à partir des années 2000, avec notamment $L^{\prime}$ 'Échelles des anges ou Dire ne suffit pas ${ }^{5}$ ). La liste des activités ne s'arrête pas là puisque, féru de psychologie et d'ésotérisme, Jodorowsky se consacre par ailleurs à l'écriture d'essais (la plupart consacrés aux relations entre les arts et le développement psychique, tels que Cabaret mystique ou encore Manuel de psychomagie ${ }^{6}$ ), pratique le tarot divinatoire et anime des "Cabarets mystiques", séances collectives mêlant psychothérapie, spectacle vivant et occultisme. Il continue aujourd'hui de développer une œuvre hétéroclite dont son dernier film, Psychomagie (2019), pourrait être le parangon, tant ce film-somme se situe à la jonction des réflexions développées dans ses essais et de ses pratiques théâtrales autant que thérapeutiques - distinction qui n'a d'ailleurs aucun sens pour Jodorowsky qui se plaît à parler de «théâtre de la guérison » pour définir toutes ses activités.

3 En dépit de la dimension foisonnante de cette œuvre, celle-ci ne fait l'objet que d'une (re)connaissance partielle. L'une des causes de cette situation peut être à chercher dans la diffusion lacunaire de ses productions, en particulier de ses textes dont beaucoup n'ont pas été traduits. Du reste, malgré une œuvre imposante, non seulement les études qui y sont consacrées sont rares, mais la plupart ne se focalisent que sur une seule pratique artistique, au premier rang desquelles le cinéma, la bande dessinée ou encore le théâtre. Bien que ces trois supports occupent une place importante dans la vie artistique de Jodorowsky, ils ne représentent quantitativement qu'une fraction d'un ensemble pléthorique. À l'instar de la partie émergée d'un iceberg, ces œuvres seraient la partie visible d'un tout largement immergé qui, comme aime à le dire l'artiste qui utilise lui-même la comparaison ${ }^{7}$, constituerait l'identité plus secrète d'un corpus multiple et varié. Par ailleurs, les œuvres de Jodorowsky semblent participer d'un réseau dont la cohérence peut se dissimuler derrière une disparité de façade. Indépendamment du genre ou du support, ses diverses créations sont en permanence traversées par une pluralité de leitmotive narratifs et formels que travaillent des effets d'échos, de renvois, de reprises d'une œuvre à l'autre, que ce soit par l'intermédiaire de symboles culturels souvent ésotériques, de matières expressives ou stylistiques, ou encore de pratiques créatorielles - autant d'échanges, de rencontres, de dialogues qui paraissent être au fondement de l'œuvre de Jodorowsky.

C'est précisément l'ambition de ce numéro que de lever ce voile qui flotte sur l'ensemble du corpus jodorowskien, et plus encore de l'envisager selon une approche transdisciplinaire prompte à mettre au jour les relations d'intermédialité, les jeux d'hybridation et d'influences esthétiques entre ses différents médiums artistiques. Trois axes peuvent dès lors être envisagés comme autant de portes d'entrée à l'étude de ces échanges artistiques. D'une part, la circulation de motifs thématiques et figuratifs, tels que ceux de l'araignée (Jules Fontaine et Marion Sergent), du corps (Sophie Pierre) ou de la rencontre (Raphaëlle Hérout), qui ne cessent d'être repris et retravaillés d'une œuvre à l'autre, s'imposant comme de véritables points d'articulation. D'autre part, ce sont les pratiques artistiques elles-mêmes qui s'influencent et s'hybrident, de la poésie, qui infuse alchimiquement l'ensemble de l'œuvre (Joy Courret), au spectacle vivant, et notamment la performance théâtrale qui, elle, informe la manière dont sont pensés, et faits, les films (Élisabeth Pouilly, Émile Combes). Enfin, certaines œuvres, et plus particulièrement celles appartenant au genre de la science-fiction, semblent entrer en connexion avec le réel en ce qu'elles interrogent directement des phénomènes culturels, tel que le jeu vidéo (Thierry Serdane) et politiques (Audrey Cavaillé), dans le 
but de brosser, pour partie, le portrait critique de leur époque, mettant ainsi en évidence le caractère actuel, voire visionnaire et prophétique, de cette œuvre plurielle.

Cette exploration des rencontres, des confrontations et des dialogues entre ces divers «actes » artistiques, selon un terme propre à l'auteur, sera introduite par Jodorowsky lui-même, qui a généreusement accepté de nous parler de son œuvre. Nul doute que son affabilité et son art de la digression contribueront eux-aussi à davantage faire émerger les liens nouveaux de cette œuvre plurielle.

\section{NOTES}

1. L'un de ces mimodrames a été adapté en bande dessinée par Olivier Boiscommun (dessin) et Jodorowsky lui-même (scénario) : Pietrolino, Les Humanoïdes Associés, 2 tomes (2007-2008).

2. Voir Fernando Arrabal, Panique. Manifeste pour le troisième millénaire, Paris, Punctum, coll. «Pour d'autres raisons », 2006.

3. Mœbius (dessin) et Alejandro Jodorowsky (scénario), L'Incal, 6 t., Paris, Les Humanoïdes Associés, coll. «Eldorado", 1981-1988; Arno (dessin) et Alejandro Jodorowsky (scénario), Les Aventures d'Alef-Thau, 8 t., Paris, Les Humanoïdes Associés, coll. "Eldorado ", 1983-1998; Juan Giménez (dessin) et Alejandro Jodorowsky (scénario), La Caste des Méta-Barons, 8 t., Paris, Les Humanoïdes Associés, 1992-2003.

4. Le Paradis des perroquets, Paris, Flammarion, 1984 ; L'Arbre du dieu pendu, Paris, Métailié, 1996.

5. L'Échelle des anges, trad. fr. Marianne Costa et Denis Patouillard-Demoriane, Paris, Albin Michel, coll. «Espaces libres ", 2005 ; Dire ne suffit pas, trad. fr. Martin Bakero, David Giannoni et Emmanuel Lequeux, Paris, Le Veilleur, 2003.

6. Cabaret mystique, trad. fr. Alex Lhermillier et Nelly Lhermillier, Paris, Albin Michel, coll. «Espaces libres », 2011 ; Manuel de psychomagie, trad. fr. Alex Lhermillier et Nelly Lhermillier, Paris, J’ai Lu, coll. « Aventure secrète », 2017.

7. Voir Alejandro Jodorowsky, «Parlons Tarot - $2 »$ : http://www.ecoledutarot.com/spip.php? article89 (dernière consultation le 06/10/2019).

\section{AUTEURS}

\section{YOANN HERVEY}

Doctorant et enseignant en études cinématographiques à l'université Paul-Valéry Montpellier 3. Également diplômé en philosophie et science politique, il termine, sous la direction de Maxime Scheinfeigel, une thèse consacrée à la philosophie du cinéma de Gilles Deleuze intitulée «Le cinéma ou l'homme réel : approche généalogique et critique du problème de la subjectivité dans les Cinéma (1. Image-mouvement ; 2. Image-temps) de Gilles Deleuze. Il codirige actuellement avec Frédéric Dallaire, Juliette Goursat et Caroline San Martin un ouvrage collectif (sur) Ce que nous 
font les films (à paraître en 2020 aux éditions Rouge Profond) et a publié plusieurs articles dans différentes revues et ouvrages collectifs, parmi lesquels Les Usages de Deleuze (dirigé par JeanPhilippe Chemineau, Ellipses, 2020, à paraître).

\section{FABIEN MEYNIER}

Doctorant au sein du centre de recherche RIRRA21 de l'université Paul-Valéry Montpellier 3. Il soutiendra en novembre 2019 une thèse intitulée « Archéologie et esthétique des lieux dans les œuvres de John Ford, Jean-Marie Straub, Danièle Huillet et Tariq Teguia. » Il a codirigé avec Nicolas Bianchi un ouvrage collectif sur l'identité et l'altérité dans les représentations de la Première Guerre mondiale qui sera publié en 2020. Il a collaboré avec les revues CinémAction, L'Entre-deux (centre de recherche Textes \& Cultures, université d'Artois) ou encore À l'épreuve (RIRRA 21).

\section{CÉLINE SATURNINO}

Docteure en études cinématographiques et auteure d'une thèse sur Gus Van Sant, elle est enseignante à l'université Paul-Valéry Montpellier III. Spécialisée en esthétique du cinéma et en analyse filmique, elle travaille sur le cinéma contemporain et nord-américain. Ses recherches portent en particulier sur l'évolution des formes filmiques ainsi que sur la relation corps/ personnage. Elle a publié notamment dans la revue Éclipses (sur Arnaud Desplechin, Jerzy Skolimowski, John Boorman, Sidney Lumet) ainsi que dans plusieurs ouvrages collectifs, dont Après Welles : influences et imitations (dirigé par Jean-Philippe Trias, éd. Deuxième Époque, 2020, à paraître), et codirige un ouvrage consacré à John Cassavetes qui paraîtra aux éditions Deuxième Époque en 2020. 